En ce siècle, où tout tend à se standardiser, à s'automatiser, où I'homme perd petit à petit, au bénéfice du mécanisme inconscient, sa valeur propre, sa supériorité sur la matière brutale, c'est un devoir, à notre avis, de dégager, quand on le peut, des manifestations de l'activité humaine qui paraissent banales parce que souvent répétées avec succès, la somme de travail, d'intelligentes recherch es et de patience qu'il a fallu à de nombreux artisans pour mener la tâche à bien.

\title{
REVUE
}

\section{LES PROGRÈS DE L'INDUSTRIE AMÉRICAINE DES COMPOSÉS DE L'ACIDE LACTIQUE}

\author{
par \\ G. GÉNIN \\ Ingénieur-chimiste E. P. C.
}

Depuis quelques années déjà, l'industrie laitière américaine a effectué d'importants travaux en vue de produire, en partant du lactose, de l'acide lactique et certains de ses composés. D'importants progrès ont été réalisés à la suite de ces travaux.

Dans le procédé mis au point dans les laboratoires de recherches de la National Dairy Products Corporation, de Baltimore, on utilise comme matière première le lait qu'on laisse sûrir de façon que le $p H$ de ce produit soit compris entre 4,6 et 5,0 , soit au point isoélectrique. On a constaté que la fermentation lactique s'effectue d'une façon plus satisfaisante lorsque les protéines ont été complètement séparées; cette séparation s'effectue en chauffant le lait à la température de $140^{\circ} \mathrm{F}$, en l'agitant et en le laissant refroidir ensuite lentement ; traitement qui a pour effet d'effectuer également une pasteurisation du produit. Après plusieurs heures de repos, on peut séparer facilement la boue de protéine qui s'est rassemblée et il est facile de séparer par décantation le sérum.

Dans le sérum ainsi obtenu, qui est presque complètement exempt de protéines, on procède à la fermentation lactique au moyen d'un organisme approprié, la fermentation demandant un temps de 10 à 24 heures. Au cours de cette fermentation, on ajoute de temps en temps au produit de l'oxyde de calcium ou tout autre composé de chaux, qui se combine à l'acide libre formé. On maintient pendant toute la durée de l'opération la température au voisinage de $100^{\circ} \mathrm{F}$. Finalement, on neutralise le liquide avec le composé de chaux et on chauffe à $180^{\circ}-220^{\circ} \mathrm{F}$. Par refroidissement, il se dépose au fond des récipients une boue de coloration blanche, 
qui est constituée principalement de différents produits azotés et d'autres impuretés; le lactate de calcium reste en solution, cette dernière est séparée par siphonage, concentrée dans les appareils travaillant sous le vide et le lactate de calcium se sépare par cristallisation.

La purification des cristaux s'effectue en les lavant dans un appareil centrifuge, on les dissout ensuite dans l'eau et on élève la température de la solution à $140^{\circ}-160^{\circ} \mathrm{F}$. A ce moment, le $p \mathrm{H}$ est réglé à 10-12 par addition de chaux; on laisse reposer pendant 2 à 3 heures et les dernières traces d'impureté se séparent sous forme de boue. On siphone la solution surnageante et on la neutralise avec une faible quantité d'acide lactique. On peut obtenir un produit encore plus pur, en clarifiant la solution au moyen de charbon et en la filtrant.

Les Sealtest System Laboratories de Baltimore ont mis au point un procédé légèrement modifié dans lequel on part de sérum préparé par le procédé usuel. Ce produit est tout d'abord neutralisé par addition de chaux, jusqu'à un $p H$ de 6,5 à 7,5 . On procède alors à la fermentation lactique, après ensemencement s'il y a lieu, en élevant la température à $110^{\circ} \mathrm{F}$, afin d'accélérer la fermentation du lactose. Comme dans le procédé précédent, on ajoute de temps en temps de la chaux qui se combine à l'acide lactique formé, et qui permet de maintenir le $p H$ à une valeur comprise entre 5,5 et 7,0 et réglable suivant le type d'organisme employé. Lorsque la fermentation est terminée, on coagule les protéines et les autres substances étrangères en élevant la température à $180^{\circ}-250^{\circ} \mathrm{F}$. et on purifie la solution surnageante par filtration.

Pour obtenir une solution absolument incolore de lactate de calcium, ce qui avait été jusqu'alors impossible, le filtrat brut est soumis à un traitement combiné au moyen d'un absorbant solide tel que charbon ou terre à foulon et d'oxyde ou d'hydrate de calcium. Ce traitement entraine une séparation rapide et complète de toutes les substances indésirables contenues encore dans le filtrat. On filtre la solution au filtre-presse, ou encore, on la passe au centrifuge et l'excès d'alcalinité est neutralisé par l'acide lactique. Les cristaux que l'on obtient après concentration dans le vide sont centrifugés et séchés.

On pourrait, par le même procédé, préparer le lactate de sodium, ainsi que les autres sels de l'acide lactique. Il a toutefois été constaté qu'il y a avantage à passer par l'intermédiaire du lactate de calcium. Pour préparer le sel de sodium, on traite une solution de lactate de calcium par du sulfate de sodium. Il y a formation de sulfate de ealcium insoluble et au contraire de lactate de sodium très soluble. La solution de ce dernier sel est passée au filtre-presse, puis concen- 
trée dans le vide. On obtient finalement non pas un corps cristallin, mais un sirop très pur qui ressemble à la glycérine.

De même, le lactate de calcium peut être employé comme intermédiaire pour la fabrication de l'acide lactique lui-même. A cet effet, le sel très pur est dissous dans l'eau chaude et la solution est acidifiée avec de l'acide sulfurique concentré ou légèrement dilué. Après passage au filtre-presse, on obtient une solution de l'acide généralement colorée par suite de la présence d'impuretés métalliques, Ces impuretés peuvent généralement être éliminées par traitement au moyen du ferrocyanure de potassium ou de tout autre réactif et au moyen de charbon.

\section{A PROPOS DE L'EMPLOI DE LA CASÉINE DANS LA FABRICATION DES MATIËRS PLASTIQUES}

Nous avons publié dans notre numéro de septembre-octobre 1937 une étude que nous avons voulu très succincte sur la fabrication des matières plastiques à base de caséine et nous avons en particulier rappelé quelques faits sur l'historique de cette industrie.

Un de nos fidèles lecteurs, M. Marc FouAssIen, nous écrit très aimablement pour nous informer, qu'à son avis, nous n'avons pas assez insisté sur l'importance essentielle, pour l'avenir de cette industrie, d'une découverte faite par un savant français, M. TrILlat, et nous communique quelques renseignements.

Devant l'intérêt que présente ce point d'histoire, nous avons demandá à notre collaborateur, M. GÉnIN, de réunir les informations qu'il a pu recueillir sur l'origine de la fabrication des matières plastiques à base de caséine, afin de compléter sa précédente note.

Il semble que c'est par un chimiste dénommé F. E. CHILds (Brevet allemand 32.293 ) que la première mention a été faite de l'emploi de la caséine du lait pour la fabrication des matières plastiques. Dans le procédé décrit par cet auteur vers 1884, on utilisait le coagulum obtenu en laissant sûrir le lait; ce coagulum était égoutté, découpé, lavé à l'eau bouillante, puis malaxé après introduction des différentes substances destinées à le rendre plus dur; finalement on le laminait ou on le moulait.

On trouve ensuite quelques années plus tard un procédé analogue décrit par O. Schoenfeld (Brevet allemand 85.886 de 1893) d'après lequel on obtenait des masses plus ou moins dures en utilisant un mélange de easéine, de matière colorante et d'oxyde de zinc, puis e'est le brevet 118.952 de C. JUNG, A. Brecher et A. KITten dans lequel on partait comme matière première de caséinate alcalin qui était, après séchage, mólangé avec des huiles sulfurées, ainsi qu'avec du caoutchouc, des résines et des matières colorantes (Brevet 